\title{
Self-Amplified Spontaneous Emission in Smith-Purcell Free-Electron Lasers
}

\author{
Kwang-Je Kim and Su-Bin Song ${ }^{1}$ \\ Advanced Photon Source, Argonne National Laboratory, Argonne, IL 60439
}

\begin{abstract}
We present an analysis of a Smith-Purcell system in which a thin current sheet of electrons moves above a grating surface in the direction perpendicular to the grating grooves. We develop a complete theory for evolution of the electromagnetic field and electron distribution in the exponential growth regime starting from the initial electron noise and the incoming amplitude. The dispersion relation for the complex growth rate for this system is a quadratic equation.
\end{abstract}

The submitted manuscript has been createc by the University of Chicago as Operator of Argonne National Laboratory ("Argonne": under Contract No. W-31-109-ENG-38 with the U.S. Department of Energy. The U.S. Government retains for itself, and others acting on its behalf, a paid-up, nonexclusive. irrevocable worldwide license in said article to reproduce, prepare derivative works, distribute copies to the public, and perform publicly and display publicly, by or on behalf of the Government.

Beginning with the work by Smith and Purcell [1], the radiation generated by an electron beam passing over a grating surface has been studied for decades [2-4]. Recently there has been a renewed interest in the Smith-Purcell system with the observation of possible exponential gain in an experiment using electron microscope beams $[5,6]$.

In this paper, we study the Smith-Purcell system in the exponential gain regime including self-amplified spontaneous emission (SASE). We study a simplified system where the electrons are line charges oriented parallel to the direction grooves and move perpendicular to the grating grooves. The electron beam is furthermore assumed to be confined to a thin sheet. The configuration is translationally invariant in the direction of the grooves, and is schematically illustrated in Fig. 1.

1 E-mail: sbsong@aps.anl.gov 
The radiation field is expanded in terms of the plane wave modes discussed by van den Berg [2]. The fundamental evanescent mode is synchronous with the electron beam and induces electron bunching through the longitudinal electric field. To take into account the interaction, the mode amplitudes are assumed to be slowly varying in the direction "perpendicular" to the wave direction. Using the boundary conditions across the electron beam and on the grating surface, the outgoing mode amplitudes are completely determined from the incoming mode amplitudes and the electron current. The evolution of the electromagnetic field and the electron distribution can be described by the coupled Maxwell-Klimontovich equations, as in the analysis of the usual free-electron laser (FEL) system [7]. The growth rate for this system satisfies a dispersion relation that turns out to be quadratic, rather than cubic as in the usual FEL process.

We obtain a formula for the growth rate that differs from that derived previously [3]. The discrepancy appears to be due to an unnecessary additional assumption employed in reference [3]. Our formula gives rise to a gain length comparable to the length of the grating for the experiment reported in reference [5].

\section{Smith-Purcell FEL Equation}

\subsection{Current}

Figure 1 shows the Smith-Purcell system studied in this paper. The surface of the metallic grating consists of a perfect conductor whose grooves are parallel and uniform in the $y$ direction. Electrons move parallel to the $z$ axis in a thin sheet along the grating surface. The current density is therefore

$$
\mathbf{J}(x, z, t)=\frac{q}{\Delta y} \sum_{i} v_{i} \delta(x) \delta\left(z-\int v_{i} d t\right) \hat{\mathbf{z}}=\frac{q}{\Delta y} \delta(x) \sum_{i} \delta\left(t-t_{i}(z)\right) \hat{\mathbf{z}},
$$

where $v_{i}$ is the velocity of the $i$ th electron, $q$ is electron charge, $\Delta y$ is the length in the $y$ direction, $t_{i}(z)=t_{i}(0)+\int\left(1 / v_{i}\right) d z$ is the time when the $i$ th electron passes through $z$, and $\hat{\mathbf{z}}$ is the unit vector in the $z$ direction. The Fourier transform of the current density is given by

$$
\mathbf{J}(x, z ; \omega)=\int \mathbf{J}(x, z, t) e^{i \omega t} d t=\frac{q}{\Delta y} \delta(x) \sum_{i} e^{i \omega t_{i}(z)} \hat{\mathbf{z}} \equiv J_{z} \hat{\mathbf{z}} .
$$

The $z$-dependent part of the phase in Eq. (2) has the average value

$$
\alpha_{0} z=\omega \frac{z}{v_{0}}=\frac{k}{\beta_{0}} z
$$




\section{DISCLAIMER}

This report was prepared as an account of work sponsored by an agency of the United States Government. Neither the United States Government nor any agency thereof, nor any of their employees, make any warranty, express or implied, or assumes any legal liability or responsibility for the accuracy, completeness, or usefulness of any information, apparatus, product, or process disclosed, or represents that its use would not infringe privately owned rights. Reference herein to any specific commercial product, process, or service by trade name, trademark, manufacturer, or otherwise does not necessarily constitute or imply its endorsement, recommendation, or favoring by the United States Government or any agency thereof. The views and opinions of authors expressed herein do not necessarily state or reflect those of the United States Government or any agency thereof. 


\section{DISCLAIMER}

Portions of this document may be illegible in electronic image products. Images are produced from the best available original document. 
where $\alpha_{0}=k / \beta_{0}, \beta_{0}=v_{0} / c$ is the normalized average velocity, $k=\omega / c$ is the wave number, and $c$ is the speed of light. We then write Eq. (2) as

$$
J_{z}(x, z ; \omega)=\delta(x) K(z, \omega) e^{i \alpha_{0} z}
$$

where

$$
K(z, \omega)=\frac{q}{\Delta y} \sum e^{i \omega\left(t_{i}(z)-z / v_{0}\right)}
$$

Since the overall phase factor $e^{i \alpha_{0} z}$ is taken out, we expect that $K(z, \omega)$ is a slowly varying function of $z$.

\subsection{Maxwell Equations}

The electric and magnetic fields in the frequency domain are defined as

$$
\begin{aligned}
& \mathbf{H}(x, z ; \omega)=\int \mathbf{H}(x, z, t) e^{i \omega t} d t \\
& \mathbf{E}(x, z ; \omega)=\int \mathbf{E}(x, z, t) e^{i \omega t} d t
\end{aligned}
$$

From the Maxwell equation it follows that the magnetic field $\mathbf{H}$ is in the $y$ direction for the present system [2] and that $H_{y}$ satisfies the following Helmholtz equation:

$$
\left(\frac{\partial^{2}}{\partial x^{2}}+\frac{\partial^{2}}{\partial z^{2}}+k^{2}\right) H_{y}(x, z ; \omega)=\frac{\partial}{\partial x} J_{z}(x, z ; \omega)
$$

The electric field is then determined by the Faraday equation:

$$
\begin{aligned}
& E_{x}(x, z ; \omega)=\frac{1}{i \epsilon_{0} \omega} \frac{\partial}{\partial z} H_{y}(x, z ; \omega) \\
& E_{z}(x, z ; \omega)=\frac{1}{i \epsilon_{0} \omega}\left[J_{z}(x, z ; \omega)-\frac{\partial}{\partial x} H_{y}(x, z ; \omega)\right]
\end{aligned}
$$

where $\epsilon_{0}$ is the vacuum dielectric constant.

\subsection{Modes}

Van den Berg has studied modes for the magnetic field $H_{y}$ in the absence of the interaction between the current and the field [2]. The plane wave solution of the Helmholtz equation is of the form [2]

$$
e^{i\left(\alpha_{n} z \pm p_{n} x\right)}
$$

where

$$
p_{n}=\sqrt{k^{2}-\alpha_{n}^{2}}
$$


For the problem we are considering here, we may take

$$
\alpha_{n}=\alpha_{0}-n G \quad\left(G=2 \pi / \lambda_{g}\right),
$$

where $\alpha_{0}$ is given in Eq. (3), $\lambda_{g}$ is the period of the grating grooves, and $n$ is an arbitrary integer.

When $p_{n}$ is real, Eq. (9) represents propagating waves. In this case we will assume $p_{n}$ to be positive. Therefore, the upper (lower) sign in Eq. (9) corresponds to an outgoing (incoming) wave to (from) $x=\infty$. However, $p_{n}$ may be imaginary. In that case, we introduce the real quantity

$$
\begin{aligned}
& p_{n}=i \Gamma_{n}, \\
& \Gamma_{n}=\left|\sqrt{\alpha_{n}^{2}-k^{2}}\right|>0 .
\end{aligned}
$$

The corresponding waves are

$$
e^{i \alpha_{n} z \mp \Gamma_{n} x} .
$$

Here the upper (lower) sign corresponds to an evanescent wave "propagating" to (from) $x=\infty$. In particular, the fundamental mode $n=0$ is evanescent:

$$
\Gamma_{0}=\frac{k}{\beta_{0} \gamma_{0}}
$$

where $\gamma_{0}=1 / \sqrt{1-v_{0}^{2} / c^{2}}$. Higher-order modes with $n<0$ are also evanescent. The mode $n>0$ may or may not be propagating. We assume that the $n=1$ mode is propagating.

The evanescent $n=0$ mode is strongly coupled to the current. Modes with $n \neq 0$ are either present as the incoming mode or generated at the grating surface. For simplicity, we consider the modes $n=0$ and $n=1$ only. Including other modes will be straightforward once the principle is understood.

In region (I) the magnetic field can be written as

$$
H_{y}^{(I)}=A_{0, I}^{+} e^{i \alpha_{0} z-\Gamma_{0} x}+A_{1}^{-} e^{i\left(\alpha_{1} z-p_{1} x\right)}+A_{1}^{+} e^{i\left(\alpha_{1} z+p_{1} x\right)} .
$$

The first term is an evanescent mode generated either directly from the electron beam or at the grating surface, the second term is an incoming mode from an external source, and the third term is a radiating mode generated at the grating surface.

In region (II) the magnetic field is

$$
H_{y}^{(I I)}=A_{0}^{-} e^{i \alpha_{0} z+\Gamma_{0} x}+A_{0, I}^{+} e^{i \alpha_{0} z-\Gamma_{0} x}+A_{1}^{-} e^{i\left(\alpha_{1} z-p_{1} x\right)}+A_{1}^{+} e^{i\left(\alpha_{1} z+p_{1} x\right)} .
$$

The first term in Eq. (16) is an evanescent mode generated directly from the electron beam, the second term is an evanescent mode generated at the grating 
surface, the third term is an incoming radiating field from an external source, and the fourth term is a radiating mode generated at the grating surface.

\subsection{Slowly Varying Amplitude Approximation}

Taking into account the interaction with the current, the amplitudes $A_{n}^{ \pm}$, except $A_{1}^{-}$, cannot be regarded as constants. The incoming wave $A_{1}^{-}$is given by an external source and may be regarded as a constant. For other waves, we expect that the amplitudes are slowly varying functions of $x$ and $z$. In order for the quantity $A_{n}^{ \pm}(x, z) e^{i(\alpha z \pm p x)}$ to satisfy the Helmholtz equation to the first order derivatives of $A_{n}^{ \pm}$, we must require

$$
\left(\alpha_{n} \frac{\partial}{\partial z} \pm p_{n} \frac{\partial}{\partial x}\right) A_{n}(x, z)=0 .
$$

Therefore, the mode amplitude $A_{n}^{ \pm}$is a function of a particular combination of $z$ and $x$ :

$$
A_{n}^{ \pm}(x, z)=A_{n}^{ \pm}\left(z \mp \frac{\alpha_{n}}{p_{n}} x\right) .
$$

\subsection{Boundary Conditions}

By using the boundary conditions at the grating surface and at the current sheet, the amplitudes of the outgoing modes can be completely determined in terms of incoming wave amplitude and surface current $K$. First, the discontinuity in $H_{y}$ between regions (I) and (II) at $x=0$ is given by the surface current $K$ :

$$
A_{0, I}^{+}(z)-\left\{A_{0}^{-}(z)+A_{0, I I}^{+}(z)\right\}=K(z) .
$$

Note that only the $n=0$ modes couple to the current since the phase velocity of these modes are the same as the average electron velocity.

Second, $E_{z}$ must be continuous across the regions (I) and (II). Using

$$
E_{z}=\frac{1}{-i \epsilon_{0} \omega}\left(\frac{\partial}{\partial x} H_{y}\right)
$$

we then have

$$
\begin{aligned}
\frac{i \alpha_{0}}{\Gamma_{0}} \frac{\partial}{\partial z} A_{0, I}^{+}(z) & -\Gamma_{0} \cdot A_{0, I}^{+}(z) \\
& =-\frac{i \alpha_{0}}{\Gamma_{0}} \frac{\partial}{\partial z} A_{0}^{-}(z)+\frac{i \alpha_{0}}{\Gamma_{0}} \frac{\partial}{\partial z} \cdot A_{0, I I}^{+}(z)+\Gamma_{0} A_{0}^{-}(z)-\Gamma_{0} \cdot A_{0 . I I}^{+}(z)
\end{aligned}
$$


Inserting Eq. (19) into (21) yields

$$
i \frac{\alpha_{0}}{\Gamma_{0}} \frac{\partial}{\partial z}\left\{K(z)+2 A_{0}^{-}(z)\right\}=\Gamma_{0}\left\{K(z)+2 A_{0}^{-}(z)\right\}
$$

whose solution is

$$
A_{0}^{-}(z)=-\frac{1}{2} K(z)
$$

because $A_{0}^{-}=0$ when $K=0$.

The third and final boundary condition is at the grating surface $x=-b$, where the incident fields are linearly related to the reflected fields:

$$
A_{m}^{+}=\sum_{n} e_{m n} A_{n}^{-}
$$

where $e_{m n}(\omega)$ is a reflection matrix of the grating. The elements of the matrix depend on the particular geometry of the grating. We have demonstrated the calculation of $e_{10}$ on a simple shallow grating case in Appendix A. More accurate calculation of these coefficients usually requires a significant amount of numerical calculation [8], and is not treated here.

Equation (24) becomes, in our problem,

$$
\begin{aligned}
A_{0, I I}^{+}\left(z-i \alpha_{0} b / \Gamma_{0}\right) e^{\Gamma_{0} b} & =e_{00} A_{0}^{-}\left(z+i \alpha_{0} b / \Gamma_{0}\right) e^{-\Gamma_{0} b}+e_{01} A_{1}^{-} \\
A_{1}^{+}\left(z+\alpha_{1} b / p_{1}\right) & =e_{10} A_{0}^{-}\left(z+i \alpha_{0} b / \Gamma_{0}\right) e^{\left(-\Gamma_{0}+i p_{1}\right) b}+e_{11} A_{1}^{-} .
\end{aligned}
$$

Inserting Eq. (25) into Eq. (19) yields

$$
A_{0, I}^{+}(z)-\left\{A_{0}^{-}(z)+e_{00} A_{0}^{-}\left(z+2 i \alpha_{0} b / \Gamma_{0}\right) e^{-2 \Gamma_{0} b}+e_{01} A_{1}^{-} e^{-\Gamma_{0} b}\right\}=K(z) .
$$

Invoking the slowly varying approximation,

$$
b\left|\frac{\partial}{\partial z} A_{0}^{-}\right| \ll \frac{\Gamma_{0}}{2 \alpha_{0}} A_{0}^{-}=\frac{1}{2 \gamma_{0}} A_{0}^{-},
$$

$A_{0}^{-}\left(z+2 i \alpha_{0} b / \Gamma_{0}\right)$ in Eq. $(27)$ can be approximated to $A_{0}^{-}(z)$. In this case, $A_{0, I}^{+}$ can be written as

$$
A_{0, I}^{+}(z)=\frac{1}{2} K(z)-\frac{e_{00} e^{-2 \Gamma_{0} b}}{2} K(z)+e_{01} A_{1}^{-} e^{-\Gamma_{0} b} .
$$

In Eq. (29), the first term corresponds to the direct field of electrons, the second term to the $n=0$ reflected mode of the grating, and the last term to the external field reflected by the grating.

The $n=1$ mode can be determined from Eqs. (23) and (26):

$$
A_{1}^{+}(z)=-\frac{e_{10}}{2} K(z) e^{\left(-\Gamma_{0}+i p_{n}\right) b}+e_{11} A_{1}^{-} e^{2 i p_{1} b}
$$


Equations (29) and (30) determine the outgoing fields $A_{0, I}^{+}$and $A_{1}^{+}$in terms of the input field $A_{1}^{-}$and the surface current $K$.

\subsection{Electron Motion}

The electron motion is affected by the longitudinal electric field at the boundary between two regions $(x=0)$ that can be obtained from Eq. (20):

$$
E_{z}(\omega)=\frac{1}{i \epsilon_{0} \omega}\left\{\Gamma_{0} A_{0 I}^{+}(z)-\frac{i \alpha_{0}}{\Gamma_{0}} \frac{\partial}{\partial z} A_{0 I}^{+}(z)\right\} e^{i \alpha_{0} z} \text {. }
$$

The second term in Eq. (31) can be ignored invoking the slowly varying approximation. From Eqs. (29) and (31), the longitudinal electric field of the $n=0$ mode at $x=0$ is obtained, in the time domain, as follows:

$$
\begin{aligned}
& E_{0 z}(0, z ; t) \\
= & \frac{1}{2 \pi i} \frac{1}{\epsilon_{0} c \gamma_{0} \beta_{0}} \int d \omega\left[\frac{1}{2} K(z)-\frac{e_{00}}{2} e^{-2 \Gamma_{0} b} K(z)+e_{01} A_{1}^{-} e^{-\Gamma_{0} b}\right] e^{-i \omega\left(t-z / v_{0}\right)} .
\end{aligned}
$$

The term $(1 / 2) K(z)$ in the square brackets of Eq. (32) gives rise to a term in the longitudinal electric field that is proportional to $\sum_{i} \delta\left(t-t_{i}(z)\right)$. This is the contribution from the self-field and should be removed in computing the force acting on the electrons.

As in conventional FEL equations of motion, we can define the electron phase and construct a set of electron equations of motion. The electron phase in this system is defined as

$$
t-z / v_{0}=\xi
$$

whose derivative is

$$
\frac{d \xi}{d z}=\left(1 / v-1 / v_{0}\right)=-\frac{\eta}{c \beta_{0}^{3} \gamma_{0}^{2}},
$$

where $\eta=\left(\gamma-\gamma_{0}\right) / \gamma_{0}$, and $\beta_{0}=v_{0} / c_{0}$.

After removing the self-field contribution from Eq. (32), the equation describing electron's energy change is

$$
\gamma_{0} m c^{2} \frac{d \eta}{d z}=\frac{q i}{4 \pi \epsilon_{0} c \beta_{0} \gamma_{0}} \int d \omega\left[e_{00} e^{-2 \Gamma_{0} b} K(z)-2 e_{01} A_{1}^{-} e^{-\Gamma_{0} b}\right] e^{-i \omega \xi}
$$

Equations (5).(34), and (35) form a complete set of Smith-Purcell FEL equations. The energy is conserved between the field and the electron bunch in this system, as shown explicitly in Appendix B. 


\section{FEL Evolution}

\subsection{Distribution Function}

We write the Klimontovich distribution function of electrons in phase space $(\xi, \eta)$ as

$$
f(\xi, \eta ; z)=\frac{1}{v_{0}\left(d N_{e} / d z\right)} \sum_{i} \delta\left(\xi-\xi_{i}(z)\right) \delta\left(\eta-\eta_{i}(z)\right)
$$

where $d N_{e} / d z$ is the line density. This distribution satisfies the continuity equation in phase space:

$$
\frac{\partial}{\partial z} f+\dot{\xi} \frac{\partial}{\partial \xi} f+\dot{\eta} \frac{\partial}{\partial \eta} f=0
$$

where dot represents the total derivative with respect to $z$. The distribution function can be separated into the smooth background $\bar{f}$ and the perturbation $\tilde{f}:$

$$
f(\eta, \xi ; z)=\bar{f}(\eta)+\tilde{f}(\eta, \xi ; z) .
$$

We have assumed that the smooth background depends only on the electron energy $\eta$. Note that Eq. (36) implies a normalization such that $\int \vec{f}(\eta) d \eta=1$.

Using Eq. (34) and Eq. (35), Eq. (37) becomes, after linearization,

$$
\frac{d f_{\omega}}{d z}+\frac{i \omega \eta}{c \beta_{0}^{3} \gamma_{0}^{2}} f_{\omega}+i\left[d_{1} K(z, \omega)-d_{2} A_{1}^{-}\right] \frac{\partial}{\partial \eta} \bar{f}=0
$$

where $f_{\omega}=\int f e^{i \omega \xi} d \xi, d_{1}=q e_{00} e^{-2 \Gamma_{0} b} / 2 \epsilon_{0} \beta_{0} \gamma_{0}^{2} m c^{3}$, and $d_{2}=q e_{01} e^{-\Gamma_{0} b} / \epsilon_{0} \beta_{0} \gamma_{0}^{2} m c^{3}$. Here the energy change of electrons is regarded as a first-order quantity. Equation (39) can be solved using the Laplace transformation:

$$
\begin{aligned}
F_{\mu}(\eta, \omega) & =\int_{0}^{\infty} e^{-\mu z} f_{\omega} d z \\
K_{\mu}(\omega) & =\int_{0}^{\infty} e^{-\mu z} K(z, \omega) d z=\frac{q v_{0}}{\Delta y} \frac{d N_{e}}{d z} \int d \eta F_{\mu}(\eta, \omega)
\end{aligned}
$$

Equation (39) is then changed into the algebraic equation

$$
\mu F_{\mu}-f_{\omega}(z=0)+\frac{i \omega \eta}{c \beta_{0}^{3} \gamma_{0}^{2}} F_{\mu}+i d_{1} \frac{\partial \bar{f}}{\partial \eta} K_{\mu}-\frac{i d_{2}}{\mu} \frac{\partial \bar{f}}{\partial \eta} A_{1}^{-}=0
$$

Thus,

$$
F_{\mu}=\frac{1}{\mu+\frac{i \omega \eta}{c 3_{0}^{3} \gamma_{0}^{2}}}\left\{-i d_{1} \frac{\partial \vec{f}}{\partial \eta} K_{\mu}+\frac{i d_{2}}{\mu} \frac{\partial \bar{f}}{\partial \eta} A_{1}^{-}+f_{\omega}(z=0)\right\}
$$


Integrating over $\eta$ yields,

$$
\begin{aligned}
\{1+ & \left.\frac{i q d_{1} v_{0}}{\Delta y} \frac{d N_{e}}{d z} \int d \eta \frac{\partial \bar{f} / \partial \eta}{\mu+\frac{i \omega \eta}{c \beta_{0}^{3} \gamma_{0}^{2}}}\right\} K_{\mu} \\
& =\frac{q v_{0}}{\Delta y} \frac{d N_{e}}{d z}\left\{\int d \eta \frac{f_{\omega}(z=0)}{\mu+\frac{i \omega \eta}{c \beta_{0}^{3} \gamma_{0}^{2}}}+i A_{1}^{-} d_{2} \int d \eta \frac{\partial \bar{f} / \partial \eta}{\mu\left(\mu+\frac{i \omega \eta}{c \beta_{0}^{3} \gamma_{0}^{2}}\right)}\right\} .
\end{aligned}
$$

Hence, the solution is the inverse Laplace transform of the above equation:

$$
K(z, \omega)=\frac{q v_{0}}{\Delta y} \frac{d N_{e}}{d z} \oint \frac{d \mu}{2 \pi i} \frac{e^{\mu z}}{D(\mu)}\left\{\sum_{i} \frac{e^{i \omega \xi_{i}}}{\mu+\frac{i \omega \eta_{i}}{c \beta_{0}^{3} \gamma_{0}^{2}}}+i A_{1}^{-} d_{2} \int d \eta \frac{\partial \vec{f} / \partial \eta}{\mu\left(\mu+\frac{i \omega \eta}{c \beta_{0}^{3} \gamma_{0}^{2}}\right)}\right\}
$$

where we have defined the dispersion function $D(\mu)$ as

$$
D(\mu)=1+\frac{i q d_{1} v_{0}}{\Delta y} \frac{d N_{e}}{d z} \int d \eta \frac{\partial \bar{f} / \partial \eta}{\mu+\frac{i \omega \eta}{c \beta_{0}^{3} \gamma_{0}^{2}}} .
$$

The first term on the right-hand side of Eq. (45) contains a sum of stochastic phase factors and describes the process of self-amplified spontaneous emission, while the second term describes the amplification of the coherent input signal $A_{1}^{-}$.

\subsection{Dispersion Relation and Exponential Gain}

The contour integral in Eq. (45) contains all singularities in the complex $\mu$ plane. In addition to poles of kinematic origin, the singularities as the solution of the dispersion relation

$$
D(\mu)=0
$$

determine the dynamics of the system [7]. For rectangular distribution,

$$
\bar{f}(\eta)= \begin{cases}\frac{1}{\Delta \eta} & \text { if } \quad|\eta|<\Delta \eta / 2 \\ 0 & \text { otherwise }\end{cases}
$$

the dispersion relation is reduced to a second-order equation

$$
\mu^{2}=\frac{q^{2} k e_{00} e^{-2 \Gamma_{0} b}}{2 \epsilon_{0} m c^{2} \Delta y \beta_{0}^{3} \gamma_{0}^{4}} \frac{d N_{e}}{d z}-\left(\frac{k \Delta \eta}{2 \beta_{0}^{3} \gamma_{0}^{2}}\right)^{2} .
$$

For vanishing energy spread $(\Delta \eta=0)$, there is a mode growing exponentially with the growth rate

$$
\mu=\frac{1}{\gamma_{0}^{2} \beta_{0}^{2}} \sqrt{\frac{2 \pi k e_{00} e^{-2 \Gamma_{0} b}}{\Delta y} \frac{I}{I_{A}}}
$$


where $I_{A}=4 \pi \epsilon_{0} m c^{3} / e=17 \mathrm{kA}$ is the Alfvén current. For a large energy spread

$$
\Delta \eta \geq \Delta \eta_{\max }=\sqrt{\frac{2 q^{2} e_{00} e^{-2 \Gamma_{0} b} \beta_{0}^{3}}{\epsilon_{0} m c^{2} \Delta y k} \frac{d N}{d z}},
$$

the two solutions of the dispersion relation are both purely imaginary corresponding to an oscillatory amplitude.

For a growing mode, the behavior of the outgoing amplitudes $A_{0}^{+}$and $A_{1}^{+}$for $x, z \rightarrow \infty$ are

$$
\begin{aligned}
& A_{0}^{+}(x, z) \propto e^{\mu\left(z+i \alpha_{0} x / \Gamma_{0}\right)} \\
& A_{1}^{+}(x, z) \propto e^{\mu\left(z-\alpha_{1} x / p_{1}\right)} .
\end{aligned}
$$

\section{Discussion}

The dispersion relation in Eq. (49) is notable since it is a quadratic equation in $\mu$ in contrast to the cubic equation in the usual FELs employing magnetic undulators [7]. This is probably due to the assumed translational invariance in the $y$ direction. A different dispersion relation, which is cubic in $\mu$, was derived in reference [3]. However they used an additional requirement that the reflection coefficient $e_{00}$ be singular "to support the synchronous wave." We find that the requirement is neither meaningful nor necessary. The correct dispersion relation is that given by Eq. (49).

An experiment on the Smith-Purcell system was recently carried out at Dartmouth college [5]. The parameters of this experiment are: $I \lesssim 1 \mathrm{~mA}, \beta_{0} \approx 0.35$, $\lambda \approx 300 \mu \mathrm{m}$. The electron beam was cylindrical with diameter $a \approx 20 \mu \mathrm{m}$. Taking $\Delta y \approx a$ and setting $e_{00} e^{-2 \Gamma_{0} b} \approx 1$, we obtain the gain length $\mu^{-1} \gtrsim 8.6 \mathrm{~mm}$. Thus the gain in the experiment is marginal since the grating length is $13 \mathrm{~mm}$.

We have analyzed an idealized Smith-Purcell system in the exponential gain regime. The theory is based on the plane wave analysis of the system in twodimensional geometry. Dispersion relation for the growth rate was derived from the coupled Maxwell-Klimontovich equation. The dispersion relation in this case is shown to be a quadratic equation, in contrast to the cubic dispersion relation of undulator FELs.

\section{Acknowledgements}

We thank Zhirong Huang for helpful discussions. This work is supported by the U.S. Department of Energy, Office of Basic Energy Sciences, under Contract 
No. W-31-109-ENG-38.

\section{Appendix A: Reflection Coefficients for Shallow Grating}

The boundary condition at the surface of the grating made of perfectly conducting material is

$$
\hat{n} \cdot \nabla H_{y}=0,
$$

where $\hat{n}$ is the unit vector normal to the grating surface. When the grating is shallow, the grating surface can be treated to first approximation as a plane mirror. Therefore $e_{m m} \simeq 1$ for all $m$. Let us illustrate the calculation of $e_{10}$. In the absence of the external radiating source, the primary mode is a simple reflection of the incident field from the electron beam $\left(e_{00} \simeq 1\right)$

$$
H_{y}=A_{0}^{-}\left[e^{i \alpha_{0} z+\Gamma_{0} x}+e^{i \alpha_{0} z-\Gamma_{0}(2 b+x)}\right]+\sum_{n \neq 0} H_{n y}^{r} .
$$

Applying the boundary condition at the surface $x=-b+X_{g} \sin (G z)$, one obtains

$$
\begin{gathered}
-i\left(\Gamma_{0}^{2} X_{g}\left(e^{i G z}-e^{-i G z}\right)+\alpha_{0} G X_{g}\left(e^{i G z}+e^{-i G z}\right)\right) B_{0} e^{-\Gamma_{0} b} e^{i \alpha_{0} z} \\
+\hat{n} \cdot \nabla \sum_{n \neq 0} H_{n y}^{r}=0,
\end{gathered}
$$

where $X_{g}$ is the amplitude of the grating. Hence, to the first order, there are two modes excited besides primary modes, i.e., $n=1$ and $n=-1$. In the case when $\alpha_{1}=\alpha_{0}-G$ is smaller than $k$, the $n=1$ mode is the only radiating mode to the first order. From Eq. (B.4), together with Eqs. (16) and (26), we can derive that $e_{10}=\frac{1}{p_{1}}\left(\Gamma_{0}^{2}-\alpha_{0} G\right) X_{g}$.

\section{Appendix B: Energy Conservation}

The energy conservation law can be expressed as Poynting's theorem

$$
\int\left[\frac{\partial u}{\partial t}+\nabla \cdot \mathbf{S}+\mathbf{J} \cdot \mathbf{E}\right] d t=0 .
$$

Because our system is localized in time, the field energy term vanishes as the integration limit is extended to infinity. Changing to the frequency domain,

$$
\int d t \nabla \cdot \mathbf{S}=\frac{1}{2 \pi} \int d \omega \nabla \cdot \mathbf{S}(\omega)=-\frac{1}{2 \pi} \int d \omega \mathbf{J}^{*}(\omega) \cdot \mathbf{E}(\omega),
$$

where $\mathbf{S}(\omega)=\mathbf{E}(\omega) \times \mathbf{H}^{*}(\omega)$. 
The energy equation for an electron is

$$
\frac{d}{d z} \gamma_{i} m c^{2}=\frac{q}{2 \pi} \int d \omega e^{-i \omega t_{i}} E_{z}(\omega)
$$

Summing up all the electrons yields from Eq. (5),

$$
\frac{d}{d z} \sum_{i} \gamma_{i} m c^{2}=\frac{q}{2 \pi} \int d \omega \sum_{i} e^{-i \omega t_{i}} E_{z}(\omega)=\frac{1}{2 \pi} \int d \omega \Delta y K^{*}(\omega) E_{z}(\omega) .
$$

Here $K$ is the surface current that gives rise to a discontinuity in $H_{y}$ between regions (I) and (II). Therefore, Eq. (B.7) becomes

$$
\frac{\Delta y}{2 \pi} \int d \omega\left(H_{y}^{(I) *}-H_{y}^{(I I) *}\right) E_{z}(\omega)
$$

From the definition of the Poynting vector, we can see that the integrand in the above equation is nothing more than the difference of the $x$ components of two Poynting vectors:

$$
-\frac{\Delta y}{2 \pi} \int d \omega\left(S_{x}^{(I) *}-S_{x}^{(I I) *}\right)
$$

Consider a volume of infinitely thin slabs at the boundary between regions (I) and (II), which is extended $\Delta y$ in the $y$ direction. Only the $x$ component of the Poynting vector will contribute to the surface integration of the given volume, because there is no finite area whose normal vector is in the direction of $z$. Thus, using the divergence theorem, the above integral becomes

$$
-\frac{1}{2 \pi} \frac{d}{d z} \int d \omega \int \mathbf{S} \cdot \hat{\mathbf{n}} d a=-\frac{1}{2 \pi} \frac{d}{d z} \int d \omega \int \nabla \cdot \mathbf{S}(\omega) d V
$$

Therefore, conservation of energy between the field and the electron bunch is shown as

$$
\frac{d}{d z}\left\{\sum_{i} \gamma_{i} m c^{2}+\frac{1}{2 \pi} \int d V \int d \omega \nabla \cdot \mathbf{S}\right\}=0
$$

\section{References}

[1] S. J. Smith, E. M. Purcell, Phys. Rev. 92 (1953) 1069.

[2] P. M. van den Berg, J. Opt. Soc. Am. 63 (1973) 689.

[3] L. Schächter and A. Ron, Phys. Rev. A 40 (1989) 876.

[4] J. H. Brownell, J. Walsh, Phys. Rev. E 57 (1998) 1075.

[5] J. Urata, M. Goldstein, M. F. Kimmitt, A. Naumov, C. Platt, J. E. Walsh, Phys. Rev. Lett. 80 (1998) 516. 
[6] J. E. Walsh, J. H. Brownell, J. Swartz, S. Trotz, M. Kimmitt, G. Doucas, R. Fernow, H. Kirk, V. Yakimenko, "Radiation induced by relativistic beams passing over a diffraction grating," these proceedings.

[7] K.-J. Kim, Nucl. Instr. Meth. A 250 (1986) 396.

[8] R. Petit (Ed.), Electromagnetic Theory of Gratings, Springer-Verlag, 1980. 
Figure

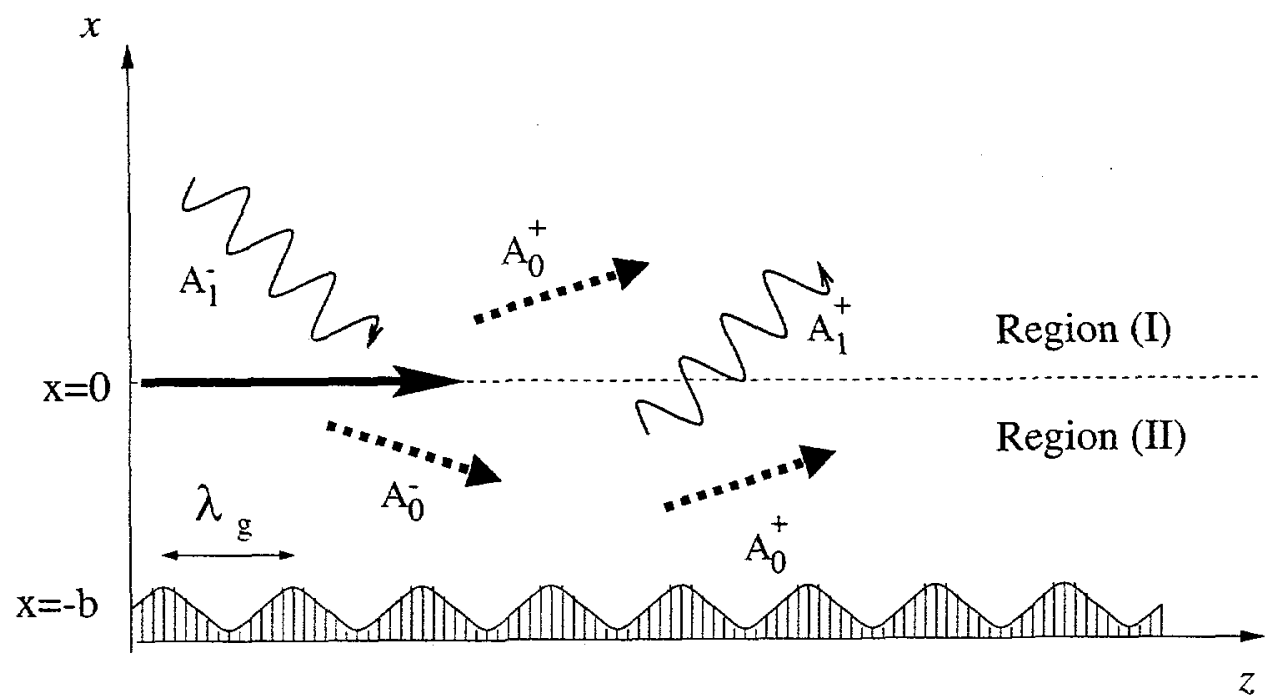

Fig. 1 Wave interaction in a Smith-Purcell system.

The system is translationally invariant in the $y$ direction (perpendicular to the page). The wave lines, the dotted lines, and the solid line at $x=0$ are respectively the $n=1$ mode, the synchronous evanescent mode, and the electron beam. The electron beam is infinitely thin in the $x$ direction. The grating surface is at $x=-b$. 